\section{Práticas de gestão em hospitais privados de médio porte em São Paulo, Brasil}

\author{
Management practices in medium-sized private \\ hospitals in São Paulo, Brazil
}

\section{Prácticas de gestión en hospitales privados de tamaño medio de São Paulo, Brasil}

\author{
Luiz Artur Ledur Brito 1 \\ Ana Maria Malik 1 \\ Eliane Brito 1 \\ Sergio Bulgacov 1 \\ Tales Andreassi 1
}

\section{Resumo}

As práticas tradicionais de gestão são, por vezes, consideradas uma condição apenas necessária para o desempenho superior. Outros recursos e competências com maiores barreiras à imitação é que seriam as potenciais fontes de vantagem competitiva. Este estudo descreve e analisa o efeito de práticas tradicionais de gestão no desempenho em hospitais de médio porte. As empresas de médio porte são aquelas em que se costuma encontrar maiores diferenças no uso das práticas, e o setor hospitalar só mais recentemente tem buscado, na área de administração, formas de desenvolver sua competitividade. De forma geral, os resultados indicam que as práticas básicas de gestão podem sim trazer diferenças de desempenho, oferecendo suporte à nova perspectiva da PBV (practice-based view). De fato, verificou-se que hospitais que apresentaram uma maior taxa de adoção de práticas possuíam maior taxa de ocupação, internações por leito e certificado de acreditação. A não adoção das práticas por hospitais de médio porte traz uma limitação de capacidade competitiva que pode ser entendida como mais um componente do custo Brasil, mas, dessa vez, um componente interno.

Administração de Serviços de Saúde; Gestão em Saúde; Hospitais

\author{
Correspondência \\ T. Andreassi \\ Escola de Administração de Empresas de São Paulo, Fundação \\ Getúlio Vargas. \\ Av. 9 de Julho 2029, São Paulo, SP 01313-902, Brasil. \\ tales.andreassi@fgv.br \\ 1 Escola de Administração de Empresas de São Paulo, Fundação \\ Getúlio Vargas, São Paulo, Brasil.




\section{Introdução}

Os hospitais brasileiros são considerados pouco eficientes, principalmente os de pequeno e médio porte 1,2,3. Em estratégia, a visão baseada em recursos propõe que a vantagem competitiva sustentada e o consequente desempenho superior ao dos concorrentes derivam da construção de recursos internos 4. Trata-se do modelo VRIO (Valor, Raridade, Imitabilidade e Organização) 5, segundo o qual uma empresa só conseguirá o desempenho superior se tiver algumas características que sejam raras, valiosas e difíceis de imitar (chamadas de "recursos") e que seja organizada em termos de políticas e processos internos para apoiar e explorar esses recursos valiosos. Por exemplo, o domínio de uma tecnologia específica que nenhuma outra empresa domine pode ser considerado um recurso. Tal modelo deriva da visão baseada em recursos e relaciona a competitividade da empresa a fatores internos, em oposição ao modelo porteriano das cinco forças competitivas, que explica a competitividade da empresa por fatores externos (concorrência, bens substitutos, fornecedores etc.).

De fato, o desempenho das empresas depende, de forma fundamental, de aspectos idiossincráticos de cada organização e de como ela reage às mudanças no ambiente externo. Estudos empíricos na área de estratégia indicam que o percentual da variância do desempenho associado a esses fatores idiossincráticos supera os $40 \%$ da variância total 6,7. As características específicas de cada empresa individual são as que mais explicam as diferenças de desempenho entre elas.

Este trabalho aborda a relação entre práticas básicas de administração, nas áreas funcionais de finanças, marketing, recursos humanos, estratégia e operações, e desempenho em hospitais privados de médio porte. Entendem-se como práticas básicas de administração aquelas utilizadas pelos gestores das áreas funcionais da administração. Por exemplo, um sistema de gestão de custos é uma prática utilizada por gestores da área de finanças; o foco no cliente é uma prática utilizada pela área de marketing; um programa para retenção de talentos é uma prática utilizada pela área de recursos humanos; um programa de melhoria contínua é uma prática utilizada pela área de operações; um evento de planejamento estratégico é uma prática utilizada pela área de estratégia. Como medidas de desempenho, este trabalho considera os indicadores taxa de ocupação, internação por leito, permanência em dias e certificado de acreditação.

A utilização dessas práticas básicas de administração não pode ser entendida como um recurso segundo a visão baseada em recursos, dado que não são raras nem difíceis de imitar. Tais práticas são ensinadas em cursos de graduação e pós-graduação de Administração, inclusive na área da gestão em saúde 8 , descritas em livros-texto e textos de divulgação, além de serem difundidas por um grande número de consultorias e instituições afins. Até pequenos empreendedores buscam o apoio de órgãos, como o Serviço Brasileiro de Apoio às Micro e Pequenas Empresas (Sebrae), para a adoção dessas práticas. Ou seja, seu uso não deveria ter relação positiva com o desempenho superior das empresas.

No entanto, Bloom \& Reenen 9,10 levantaram um debate ao investigar empiricamente o tema. Eles identificaram que empresas com menor intensidade no uso dessas práticas também apresentam pior desempenho financeiro, o que pode indicar que elas também são importantes para o desempenho superior, além do fato de as empresas possuírem recursos. O uso menos intenso das práticas, no estudo desses autores, está associado a empresas médias, de origem familiar e em países emergentes, embora exista variabilidade também em países desenvolvidos. Empresas multinacionais, contudo, apresentam utilização mais elevada das práticas gerenciais mesmo quando atuam em países emergentes. A abrangência das práticas estudadas, sua medição e análise podem ser fontes de vários questionamentos, mas o resultado desafia a teoria estabelecida. Uma resposta inicial a essa problemática pode ser encontrada no artigo de Bromiley \& Rau 11, publicado no periódico mais influente de estratégia no final de 2014. Nesse artigo, os autores questionam as limitações das teorias atuais e propõem os princípios do que chamam de practice-based view (PBV) como outra perspectiva teórica para explorar esses fenômenos.

Ressalta-se também a importância de focalizar o setor da saúde, conhecido, em geral, por ser um late adopter 12,13 do conhecimento gerencial nas suas organizações. No Brasil, tal fenômeno é ainda mais presente, tendo, em vista, a alta prevalência de médicos, sem formação na área de gestão, administrando serviços do setor. Além disso, o gasto per capita em saúde no Brasil, em paridade de poder de compra (PPP), foi de US\$ 900 em 2009, enquanto que, nos Estados Unidos, tal valor foi de US\$ 8.000 e, no Reino Unido, um dos benchmarks do setor, US\$ 3.500 14. Tais números demonstram ainda 
mais a necessidade de uma gestão eficaz dos recursos, dado o baixo volume dos gastos em saúde no Brasil frente aos países mais desenvolvidos. Várias empresas (públicas e privadas) de serviços de saúde passam por problemas de financiamento, potencializados pela falta de gestão eficaz.

Com base nesse contexto, o trabalho se propõe a analisar os efeitos das práticas tradicionais de gestão no desempenho em hospitais de médio porte. Para tanto, foram estabelecidas quatro questões específicas: (1) Quais as características das práticas gerenciais dos hospitais por área funcional de marketing, operações, recursos humanos, estratégia e finanças? (2) Como podem ser classificados os hospitais segundo nível de utilização das práticas de gestão? (3) Quais as práticas de gestão mais utilizadas por área funcional? (4) Qual a relação entre o nível de utilização das práticas de gestão e o desempenho médio dos hospitais?

Simon 15 estudou características das empresas alemãs que podem ser adaptadas ao contexto brasileiro, tendo, em vista, a competitividade de empresas médias nacionais, geralmente de origem familiar. Ele encontrou que empresas alemãs de pequeno e médio porte estão em mercados fragmentados e maduros, não operando em indústrias modernas como computadores, telefones móveis ou equipamentos eletrônicos. No Brasil, o tema é ainda inexplorado e constitui-se numa ameaça à competitividade das médias empresas frente à crescente presença de multinacionais, produtos e serviços importados. O uso menos intenso de práticas de gestão conhecidas pelas empresas médias nacionais pode ser visto como outro elemento do "custo Brasil", dessa vez, interno, em oposição à apresentação frequente das limitações externas da logística, burocracia, carga de impostos, educação etc.

No caso dos hospitais, pode-se destacar que, desde o início da década de 1990, Cleverley \& Harvey 16 já apontaram para a relação entre práticas estratégicas e funcionais e sua relação com o desempenho competitivo. Identificaram relações entre controle de custos, participação em segmentos de mercado, política e diversificação de fontes de financiamento. Qin 17 analisa a competitividade dos hospitais chineses e sugere que comparações com o mercado hospitalar mundial, por meio da ampliação da capacidade de planejamento e de sistemas informacionais mais efetivos, contribuem significativamente para o desempenho competitivo.

No Brasil, Pereira et al. 18 demonstram a efetividade das decisões e ações gerenciais por meio do uso adequado de instrumentos de gestão e sistemas de informação, inclusive para a melhora da qualidade dos diagnósticos médicos e, consequentemente, para a competitividade entre as organizações de saúde.

A investigação de Souza et al. ${ }^{19}$, sobre controle da gestão hospitalar com vistas à eficiência dos hospitais de Minas Gerais, concluiu que baixos índices de complexidade assistencial e número de leitos estão relacionados a desenvolvimento gerencial incipiente. Os aspectos mais relevantes foram controle, falta de sistemas de informações relacionados a custeio, precificação e tomada de decisão, avaliação de desempenho e ausência de ferramentas que evidenciem capacidade de monitoramento e de avaliação de resultados.

Esta pesquisa pretende avançar na exploração desse tema, analisando os efeitos das práticas tradicionais de gestão no desempenho e endereçando algumas limitações dos trabalhos de Bloom \& Reenen ${ }^{9}$, como a definição das práticas, o foco setorial e o uso de métodos complementares qualitativos.

\section{Referencial teórico}

Este trabalho tem como pressuposto que o desempenho dos hospitais depende de suas capacidades gerenciais. A visão baseada em recursos 20 propõe que os recursos e as competências considerados raros e que não conseguem ser imitados ou substituídos pelos concorrentes criam diferenças sustentadas de desempenho. A visão baseada em recursos se origina no final dos anos 1980 com as contribuições clássicas de vários autores 21,22,23 e se desenvolve na década de 1990 e início do século XXI, para se tornar uma perspectiva teórica dominante 24.

Apesar da prescrição da visão baseada em recursos, de que práticas facilmente imitáveis não são suficientes para gerar vantagem competitiva sustentável, estudos em várias áreas funcionais da Administração sugerem que a excelência na gestão pode contribuir para o desempenho das organizações. Por exemplo, o impacto positivo do marketing para o desempenho da empresa 25,26,27,28 vem sendo evidenciado desde a década de 1980. Webster Jr. ${ }^{29}$ afirma que o marketing ajudou as empresas ameri- 
canas a ganharem posições dominantes na economia mundial, e que, no momento em que deixaram esse foco, elas perderam vantagem competitiva.

Atualmente, tem sido comum, no discurso de dirigentes de hospitais públicos e privados, nacional e internacionalmente, a menção ao cuidado centrado no paciente. Trata-se de tema que entrou em voga há alguns anos, como um dos pilares das iniciativas de melhoria da qualidade e da segurança da prestação de serviços de forma geral e dos cuidados à saúde no campo específico, embora não seja fácil observar sua implantação na prática. Nos Estados Unidos, o Beryl Institute tem se dedicado à divulgação dessas iniciativas 30,31 já estimuladas pelo Center for Medicare and Medicaid Services (CMS), o grande financiador público da assistência estadunidense e presentes em hospitais de referência. No Brasil, a presença de unidades voltadas especificamente à experiência do paciente ainda está restrita a poucos serviços e, embora encontrada nos discursos dos executivos hospitalares, ainda não aparece como prioridade 32 .

Embora esse discurso tenha uma orientação mercadológica, ele abrange ainda outras áreas funcionais, cuja configuração representa um dos pilares para a melhoria da saúde, da qualidade e da segurança do serviço prestado. No caso do setor público, além das acreditações, até mesmo programas do Ministério da Saúde (no caso, humanização) se ocupam desse aspecto 33,34. Outros autores também constataram relação positiva entre desempenho e práticas de marketing 35,36 ou orientação para o mercado 37 ou, ainda, orientação para o cliente ${ }^{38}$.

Barlett \& Goshall 39 apresentam algumas características específicas do que chamaram de "late movers”. Essas são empresas situadas em países emergentes, que estão desenvolvendo negócios globais tardiamente, quando comparadas a concorrentes de outros países mais industrializados. Os autores listam algumas questões distintas daquelas propostas por Simon 15: a necessidade de criação de produtos competitivos globalmente; a dificuldade de compreensão de questões culturais em mercados além do local; e a busca por produtos de maior valor agregado. Nesses casos, estão presentes aspectos relativos à qualidade de produto ou serviço, nos quais a área de operações terá um papel central, como é o caso de hospitais.

A literatura tem destacado a importância do esforço interfuncional, incluindo manufatura, marketing, pesquisa e desenvolvimento (P\&D) e fornecimento, dentre outras. Na área da saúde, isso começa a ser chamado de gestão da produção, inovação e logística. $\mathrm{O}$ advento da produção enxuta (lean) tem mostrado, nas últimas décadas, a importância da participação da área de produção/operações no processo estratégico das empresas, lembrando que, no Brasil, o início de sua utilização em saúde é posterior a 2010 e, em geral, restrita ao setor privado. O mesmo pode ser esperado quando as empresas competem com grandes empresas globais. Nesse caso, como mencionado por Simon 15, o conhecimento mais próximo das diferentes necessidades de mercado, a coordenação das operações, $\mathrm{P} \& \mathrm{D}$ e vendas e as pressões dos concorrentes levariam a uma relação mais próxima entre produção/operações e outras áreas funcionais da empresa. Ward et al. 40 e Hausman et al. 41 mostraram a importância de uma orientação interfuncional para o desempenho da empresa. Em ambos os estudos, decisões interfuncionais são identificadas como uma das questões centrais para o aumento da competitividade.

\section{O setor hospitalar no Brasil}

No Brasil, as estatísticas vêm mostrando diminuição no número de hospitais, nos últimos anos, segundo os dados do Cadastro Nacional de Estabelecimentos de Saúde (CNES. http://cnes.datasus. gov.br, acessado em Jul/2015). Em outubro de 2013, eles totalizavam 6.744, divididos em 5 portes: até 19 leitos - porte 0; de 20 a 49 leitos - porte I; de 50 a 149 leitos - porte II; de 150 a 299 leitos porte III; 300 e mais leitos - porte IV. Os leitos no país seguem a seguinte distribuição: 22,6\% (porte 0), 34,4\% (porte I); $31 \%$ de porte II; 9,3\% de porte III e apenas 2,7\% de porte IV. Portanto, $57 \%$ dos hospitais brasileiros têm até 50 leitos, e $88 \%$, até 150 leitos, sendo que a Região Sudeste compreende $35,72 \%$ do total de hospitais.

O número de leitos no país permite entender a disponibilidade dos serviços para a população por meio do indicador leitos/população. Internacionalmente, o valor desse indicador também vem diminuindo devido a vários fatores, desde a mudança no perfil epidemiológico da população até a disponibilidade de dispositivos de diagnósticos e de drogas. No Brasil, o número de leitos variou de um número absoluto de 475.453, em 2008, para 478.018, em 2012, ao mesmo tempo em que a 
população aumentou. Seria esperado que a redução na necessidade por número de leitos ocorresse em função também do aumento da eficiência de sua utilização, com possibilidade de aumento na sua taxa de ocupação e redução nas médias de permanência, como tem sido observado em outros países.

Dados de 2012 do CNES mostram, porém, que os valores para esses indicadores são diferentes em função do tipo de hospital. Para uma taxa de ocupação preconizada de acima de $85 \%$ em condições de eficiência, nos públicos, ela era de 51,2\%; nos privados sem finalidade lucrativa, de 50,5\%, e, nos privados com finalidade lucrativa, de $41 \%$. Esses dados apontam para uma ineficiência pouco aceitável, principalmente considerando uma média nacional, numa realidade em que há alguns hospitais com cerca de $100 \%$ de ocupação. O fato de hospitais públicos terem, em média, taxas de ocupação mais elevadas, pode-se dar pelo fato de que esses têm, com frequência, principalmente no nível municipal, maior disponibilidade de recursos, desde humanos até materiais. O CNES mostra que a taxa de ocupação também aumenta com o porte dos hospitais, sendo mais elevada nos hospitais de portes III e IV (entre 63\% e 64\%) que nos 0, I e II (respectivamente, de 16\% a 44\%). Órgão internacional de pesquisa e consultoria na área hospitalar 42 afirma que, num hospital de 600 leitos, mais de 21 mil leitos-dia poderiam ser liberados por ano, com a redução de 10\% do desperdício em sua utilização.

O financiamento hospitalar no Brasil se dá, no caso dos hospitais públicos, diretamente pelo Sistema Único de Saúde (SUS); no caso dos filantrópicos, pelo SUS (diretamente por procedimentos e/ou por renúncia fiscal), por operadoras privadas de saúde e eventualmente por pagamento direto dos usuários ou das empresas onde trabalham. No caso dos hospitais com finalidade lucrativa, o setor público entra com algum tipo de subsídio indireto, e o restante ocorre como nos hospitais sem finalidade lucrativa.

Em geral, a gestão das organizações do setor no Brasil ainda está em processo de profissionalização. Um exemplo muito citado é o da certificação internacional. Na França, ela é obrigatória, enquanto que, nos Estados Unidos, ela é voluntária. Porém, quando o hospital não tem o certificado da avaliação externa, ele recebe menos financiamento. No Brasil, da mesma forma, trata-se de um processo voluntário, não ligado ao governo.

Dados disponíveis nos sítios de Internet dos modelos de acreditação em atividade no Brasil (ONA - Organização Nacional de Acreditação; JCI - Joint Commission International; AC - Accreditation Canada; NIAHO - National Integrated Accreditation for Healthcare Organizations) demonstram que, decorridos 15 anos desde a acreditação do primeiro hospital no país, o número de hospitais acreditados não chega a 5\%, podendo indicar uma falta de preocupação com a avaliação externa.

\section{Método}

Esta pesquisa foi estruturada em duas fases. A primeira, de cunho qualitativo, consistiu no estudo de três casos 43,44 do setor hospitalar, identificados com base nos contatos e experiências dos autores do trabalho. O objetivo dessa fase foi identificar práticas gerenciais utilizadas em hospitais e estruturar o questionário a ser utilizado na fase quantitativa. A Tabela 1 descreve o perfil dos três hospitais pesquisados.

Uma das definições relevantes para a condução da pesquisa foi a seleção de hospitais de médio porte como objeto, por sua representatividade em termos de gestão. Para esse fim, foi utilizada a classificação por número de leitos, já mencionada anteriormente: até 19 leitos - porte 0; de 20 a 49 leitos - porte I; de 50 a 149 leitos - porte II; de 150 a 299 leitos - porte III; 300 e mais leitos - porte IV. Nesta pesquisa, foram considerados apenas os hospitais de porte médio, correspondentes à categoria II. O Hospital B, por ter 150 leitos e estar no limite entre os portes II e III, acabou sendo considerado também como hospital de médio porte.

\section{Coleta dos dados qualitativos}

O relacionamento dos pesquisadores na área hospitalar facilitou o acesso às organizações pesquisadas e aos seus gestores. Em cada caso, foram entrevistados gestores principais da empresa e responsáveis pelas diferentes áreas funcionais. Para melhor compreender a organização C, que atua em uma categoria de negócio bem específica, foi entrevistado também o gestor de outra empresa que atua no mes- 
Tabela 1

Perfil dos hospitais pesquisados na análise qualitativa.

\begin{tabular}{|c|c|c|c|c|c|}
\hline Hospital & $\begin{array}{l}\text { Número de } \\
\text { funcionários }\end{array}$ & $\begin{array}{l}\text { Número } \\
\text { de leitos }\end{array}$ & $\begin{array}{l}\text { Número de } \\
\text { leitos de UTI }\end{array}$ & $\begin{array}{l}\text { Acreditação } \\
\text { internacional }\end{array}$ & Outras informações \\
\hline$A$ & 400 & 90 & 19 & Sim & $\begin{array}{l}\text { Possui um comitê de qualidade; realiza diversos treinamentos } \\
\text { mensais via Instituto de Ensino e Pesquisa }\end{array}$ \\
\hline B & 541 & 150 & 42 & Sim & $\begin{array}{l}\text { Gestão profissionalizada e atendimento de alta complexidade; } \\
\text { estrutura organizacional contempla as áreas da Administração }\end{array}$ \\
\hline C & 70 & 47 & - & Não & $\begin{array}{l}\text { Investe na formação de equipe multiprofissional, demandando um } \\
\text { trabalho fundamentalmente de equipe, não apenas médico }\end{array}$ \\
\hline
\end{tabular}

mo setor. O número de entrevistas variou de hospital para hospital, pois dependeu da qualidade das informações fornecidas pelos entrevistados. Ao todo, foram feitas nove entrevistas, incluindo a que foi realizada com o concorrente da organização C. Cada entrevista foi conduzida por dois membros da equipe de pesquisa. Todas as entrevistas foram gravadas e transcritas para análise posterior. Cada caso resultou de três a cinco horas de gravação. Considerando a necessidade de triangular os dados 45 , além das entrevistas, foram feitas observações com anotações sobre as condições das áreas de trabalho e dos seus processos, bem como a consulta a dados secundários em sistemas administrativos, registros e relatórios de trabalho.

Os dados foram coletados por meio de entrevistas com um roteiro composto por três blocos. Um bloco inicial pedia informações sobre a empresa, tais como ano de fundação, número de funcionários etc. Um segundo bloco endereçava questões sobre as práticas gerenciais nas áreas de marketing, operações, finanças, estratégia e recursos humanos. Por fim, um terceiro bloco, de cunho mais genérico, abrangia os temas inovação, tomada de decisão, governança e modelo de negócio. As questões do segundo e terceiro blocos foram formuladas com base em uma revisão de literatura específica, bem como pela experiência dos autores da pesquisa na área. A intenção principal das entrevistas foi aprender com os casos para definir o questionário de coleta para a fase seguinte da pesquisa. A análise foi realizada por equipe composta de pesquisadores da área de Administração de Empresas de diferentes especialidades, incluindo a área de Gestão da Saúde.

\section{Coleta dos dados quantitativos}

A segunda fase da pesquisa compreendeu um levantamento de dados junto a gestores de hospitais privados de médio porte localizados no Estado de São Paulo, Brasil, usando um questionário estruturado, construído para esse fim a partir dos achados da análise qualitativa. Justifica-se a escolha do Estado de São Paulo para esta pesquisa exploratória pelo fato de que se imagina que nesse a gestão esteja mais profissionalizada. Um dos indícios para isso é que, embora no Brasil menos de 5\% do total dos hospitais estejam acreditados, em São Paulo, essa porcentagem chega a $13 \%$.

A amostra foi definida a partir da lista das empresas associadas ao Sindicato dos Hospitais de São Paulo (SindHosp). Quatrocentos hospitais de diferentes portes faziam parte dessa lista; dos quais, 195 possuíam entre 20 e 110 leitos. Os 195 hospitais da base foram contatados por pesquisadores treinados para a coleta, e $47(24,1 \%)$ responderam a pesquisa, que foi realizada por telefone.

Para cada uma das práticas, os entrevistados avaliavam o grau de utilização da prática segundo a escala apresentada a seguir: 1 - praticamente não utiliza; 2 - usa algumas características da prática; 3 - usa várias características da prática; 4 - usa a maioria das características da prática; 5 - usa a prática de forma plena.

Foi empregada a abordagem de agrupamentos - técnica K-means do IBM SPSS 21 (IBM Corp., Armonk, Estados Unidos) - a fim de agrupar os hospitais de acordo com o nível de utilização das práticas de gestão. A partir da aplicação de tal técnica, foram identificados três agrupamentos, e, 
para cada agrupamento, foram calculados a média de utilização das práticas, o perfil dos hospitais e os indicadores.

\section{Resultados e discussão}

\section{Análise qualitativa}

A análise dos dados identificados na fase qualitativa, realizada por meio da triangulação das práticas dos hospitais coletadas nas entrevistas, de observações e da análise de documentos dos hospitais, tais como os relatórios de gestão, demonstra o contexto organizacional, as práticas e os instrumentos de gestão de cada uma das áreas funcionais. Tal análise encontra-se resumida na Tabela 2. Em relação a recursos humanos, foram identificadas as práticas de gestão de equipes, gestão de pessoas, clima de trabalho, retenção de talentos, flexibilidade na organização e clareza de responsabilidade. De fato, a retenção dos recursos humanos qualificados é um ponto crítico no setor hospitalar, razão pela qual as instituições analisadas desenvolvem práticas visando aumentar a satisfação dos funcionários e, consequentemente, diminuir o turnover.

As práticas de marketing evidenciadas foram foco no cliente e monitoramento do mercado. Entre todas as áreas funcionais analisadas, as práticas de marketing foram as menos desenvolvidas e aplicadas pelos hospitais. Não há monitoramento de mercado, acompanhamento dos concorrentes ou processos voltados para a prospecção de novos clientes. Em nenhum dos hospitais analisados, foi verificada uma estratégia mercadológica consistente, sendo muito mais reativa do que pró-ativa. Fluxo de trabalho, melhorias contínuas, monitoramento do desempenho, solução de problemas, padronização, protocolos e gestão à vista ou transparência de dados foram as práticas de operações identificadas. Talvez pelo alto custo dos insumos hospitalares e a manipulação altamente precisa, dado que um erro ou a falta de um medicamento pode por a vida do paciente em risco, as práticas de operações são bastante desenvolvidas. Os estoques são informatizados, os fornecedores, padronizados, há busca por certificação nacional e internacional, o que certamente exige o atendimento de padrões de qualidade.

Em finanças, as práticas observadas foram a própria existência de uma gestão financeira efetiva e a gestão de custos. Os softwares de ERP ou gestão financeira aplicados ao setor hospitalar já estão há tempos no mercado, razão pela qual os casos analisados já o adotam. Por fim, quanto às práticas de estratégia, ressaltam-se o planejamento estratégico e a inovação. A utilização de tais práticas variava bastante dependendo da instituição, embora todas as utilizassem. Enquanto que, em um dos hospitais, o planejamento era bastante centralizado, no outro, era participativo, com a inclusão de todos os funcionários. Já no terceiro, a centralização era relativa.

Em relação aos indicadores de desempenho, a pesquisa qualitativa mostrou que os principais indicadores utilizados pelos hospitais eram a taxa de ocupação, as internações por leito e a permanência do doente. Incluímos também o fato de o hospital ser acreditado ou não como um indicador de sua excelência.

Após a definição conceitual de cada um dos termos identificados, foi possível caracterizar as variáveis (práticas) investigadas no levantamento da fase quantitativa, descritos na Tabela 3.

\section{Análise quantitativa}

A amostra pesquisada foi de 47 hospitais que se dispuseram a responder o questionário, em uma população de 195 hospitais privados dos portes considerados no Estado de São Paulo, segundo dados fornecidos pelo SindHosp. Alguns dados do perfil dos hospitais pesquisados estão apresentadas na Tabela 4.

Os escores de avaliação de cada uma das práticas de gestão analisadas estão apresentados na Tabela 3. Os resultados indicam um nível intermediário de utilização das práticas de gestão, já que as médias ficaram, em geral, entre 3 e 4 , considerando notas de 1 a 5 . Observa-se, porém, variabilidade bastante grande, evidenciada pelos valores máximo e mínimo e pelo desvio padrão, que ficou em torno de 1 para a maioria das práticas. Essa variabilidade confirma os resultados encontrados nos estudos anteriores, no ambiente hospitalar e em empresas em geral 9,10. 
Tabela 2

Práticas gerenciais por área funcional.

\begin{tabular}{|c|c|c|c|}
\hline Área & Hospital A * & Hospital B ** & Hospital C *** \\
\hline Operações & $\begin{array}{l}\text { Processo de compras centralizado. Médicos } \\
\text { possuem grande poder na avaliação da } \\
\text { qualidade e seleção dos produtos. } \\
\text { Farmácia tem gestão informatizada } \\
\text { permitindo o controle do estoque. } \\
\text { Há um comitê de padronização de } \\
\text { medicamentos. } \\
\text { O hospital é membro de um organismo } \\
\text { americano que atua mundialmente na } \\
\text { avaliação dos hospitais e suas melhores } \\
\text { práticas. }\end{array}$ & $\begin{array}{l}\text { Fornecedores são gerenciados em } \\
\text { três categorias: medicamentos } \\
\text { (aproximadamente } 600 \text { itens, adquiridos } \\
\text { via pregão eletrônico); materiais OPME } \\
\text { (órteses, próteses e materiais especiais) } \\
\text { e contratos administrativos (limpeza, } \\
\text { alimentação, entre outros). } \\
\text { Busca-se a redução do número de } \\
\text { fornecedores por meio do estabelecimento } \\
\text { de parcerias a fim de conciliar eficiência e } \\
\text { qualidade. } \\
\text { Uso de prontuários eletrônicos integrados } \\
\text { contribui para a melhoria da qualidade e } \\
\text { confiança no serviço prestado. } \\
\text { Busca por certificação nacional e } \\
\text { internacional impulsionou a mudança } \\
\text { para o modelo de gestão por processos. } \\
\text { As melhores práticas são avaliadas e } \\
\text { incorporadas com as sugestões dos } \\
\text { colaboradores. }\end{array}$ & $\begin{array}{l}\text { A área de suprimentos ainda está se } \\
\text { estruturando na empresa. Não há } \\
\text { sistemas muito sofisticados de compras } \\
\text { e gestão de estoque. As próprias } \\
\text { características do hospital (baixo } \\
\text { número de leitos, maior uniformização } \\
\text { do estado dos pacientes) reduzem } \\
\text { significativamente a demanda por } \\
\text { medicamentos, se comparada aos } \\
\text { hospitais A e B. O hospital tem investido } \\
\text { bastante na qualidade assistencial, } \\
\text { medida por meio dos indicadores } \\
\text { clássicos de assistência, como } \\
\text { quedas do leito, perda de sondas e } \\
\text { broncoaspiração. } \\
\text { A empresa tem empregado recursos } \\
\text { para se qualificar e obter acreditação. } \\
\text { O planejamento integrado é feito por } \\
\text { meio de protocolos - de captação de } \\
\text { pacientes, de admissão de pacientes, de } \\
\text { tratamento, dentre outros. }\end{array}$ \\
\hline $\begin{array}{l}\text { Recursos } \\
\text { humanos }\end{array}$ & $\begin{array}{l}\text { O hospital faz anualmente uma pesquisa de } \\
\text { clima. Pontos de atenção são: rotatividade } \\
\text { e demissão. Questões norteadoras são: } \\
\text { orgulho do funcionário em trabalhar } \\
\text { na instituição e a indicação do hospital } \\
\text { como alternativa de emprego. Ponto } \\
\text { de destaque: a liderança, que contribui } \\
\text { para o estabelecimento de um clima } \\
\text { "bom". Há práticas integradas de recursos } \\
\text { humanos, como avaliação de desempenho, } \\
\text { treinamento e remuneração. }\end{array}$ & $\begin{array}{l}\text { Baixa oferta de mão de obra qualificada } \\
\text { é um problema crônico, que atinge } \\
\text { especialmente a categoria dos técnicos de } \\
\text { enfermagem, caracterizada por alto índice } \\
\text { de turnover. } \\
\text { Para aumentar o interesse e a retenção na } \\
\text { carreira em todos os níveis, há um sistema } \\
\text { de avaliação de desempenho semestral, } \\
\text { com participação nos resultados e um } \\
\text { plano de cargos e salários. As equipes de } \\
\text { atendimento são gerenciadas com a ajuda } \\
\text { de um sistema de informação que permite } \\
\text { atendimento imediato aos eventos críticos } \\
\text { da área. Essa atuação aumenta a confiança } \\
\text { do corpo clínico e técnico na gestão do } \\
\text { hospital. A gestão de recursos humanos } \\
\text { é acompanhada por indicadores como } \\
\text { absenteísmo, rotatividade, acidentes de } \\
\text { trabalho, exames periódicos, entrevistas de } \\
\text { desligamento e treinamentos dedicados. }\end{array}$ & $\begin{array}{l}\text { Os processos de captação, contratação, } \\
\text { treinamento e remuneração de pessoas } \\
\text { ainda estão sendo estruturados. Apenas } \\
\text { em maio de 2013, contrataram um } \\
\text { profissional de recursos humanos } \\
\text { para tratar dessas questões. No que } \\
\text { diz respeito à gestão de equipes, a } \\
\text { organização tem se diferenciado no } \\
\text { mercado, já que é a única que investe } \\
\text { em reabilitação (concorrentes atendem } \\
\text { pacientes de longa permanência usando } \\
\text { apenas tratamentos paliativos, que } \\
\text { demandam intensidade de trabalho, } \\
\text { em geral, não qualificado). As equipes } \\
\text { multifuncionais têm a missão de } \\
\text { reabilitar o paciente e sua atuação é } \\
\text { mais específica e intensa. A clínica tem } \\
\text { uma estrutura horizontal de equipe, } \\
\text { e a diferenciação se dá pelo foco na } \\
\text { realização. }\end{array}$ \\
\hline
\end{tabular}

(continua) 
Tabela 2 (continuação)

\begin{tabular}{|c|c|}
\hline Área & Hospital A * \\
\hline Estratégia & $\begin{array}{l}\text { Processo de planejamento estratégico } \\
\text { centralizado por meio de um comitê. } \\
\text { Diretores do hospital têm autonomia para } \\
\text { encaminhar a discussão nesses comitês. } \\
\text { Durante dois anos, a empresa utilizou o } \\
\text { balanced scorecard para direcionar as ações, } \\
\text { avaliar desempenho e escolher médicos por } \\
\text { meio de indicadores. "As pessoas precisam } \\
\text { parar de escolher o médico porque ele é } \\
\text { famoso, simpático e tal e começar a escolher } \\
\text { pelos predicados que ele tem. Eu começo a } \\
\text { incentivar as pessoas a perguntarem para o } \\
\text { médico quantas cirurgias ele fez desse tipo, } \\
\text { qual a mortalidade dele, qual a complicação. } \\
\text { [...] Quando a gente entra nesses bancos de } \\
\text { dados, envia os nossos resultados para lá e } \\
\text { recebe um relatório de volta é exatamente } \\
\text { para mostrar isso". A instituição americana } \\
\text { à qual o hospital é filiado também fornece } \\
\text { informações sobre análise de mercado e } \\
\text { aponta tendências, que são importantes }\end{array}$ \\
\hline
\end{tabular}

Finanças

O orçamento é uma prática ainda recente na gestão hospitalar, porém o grupo elabora orçamento há alguns anos. Atualmente, existe a preocupação de os hospitais próprios de operadoras serem tão eficientes quanto os da rede credenciada. Isso forçou os gestores a trabalharem com orçamento e a planejarem financeiramente o desempenho do hospital. A organização possui um sistema de gestão financeira, conseguindo efetivamente dados sobre lucratividade, custos, retorno de investimento ou custo/benefício dos equipamentos adquiridos. "Tem tudo detalhado. É um sistema já. [...] é tudo lançado, tem uma conta gigante de orçamento, tudo especificado por departamento. [...] Eu sei do fluxo de caixa semanalmente como está, tanto o fluxo de caixa quanto a minha despesa".

\section{Hospital B **}

Hospital C***

A partir da análise das condições concorrenciais, a proposta de diferenciação estratégica firma-se na oferta de serviço corporativo de alta qualidade. Busca-se o ganho em escala a fim de consolidar sua posição no mercado local por meio da expansão da oferta de serviços de Oncologia mais evoluídos e pelos investimentos em procedimentos de diagnósticos por imagem. O planejamento estratégico é participativo, realizado a cada dois anos e revisado semestralmente. A Análise SWOT (Forças, Oportunidades, Fraquezas e Ameaças, em português) é realizada com todos os funcionários de todos os níveis. As metas estratégicas são verificadas trimestralmente. Percebe-se inclinação para a inovação com a adoção de tecnologias emergentes e uma postura colaborativa com os fornecedores. A estratégia de abertura a fusões e aquisições poderá contribuir para os desafios de endividamento do grupo.

O hospital conta com um sistema de gestão financeira que permite avaliar adequadamente custos, lucratividade e diferentes dimensões operacionais, tais como margem de comercialização de materiais, receita paciente/dia, receita operacional diária, receita agregada por funcionário, entre outros. A gestão contábil-financeira é auditada externamente. Existe sistema de informações, de categoria ERP (Enterprise Resource Planning). Há a previsão da implantação iminente de um novo sistema de gestão, de menor complexidade e maior integração, como aposta para a melhoria da qualidade das informações. A disponibilidade de prontuário eletrônico integrado é a principal vantagem do novo sistema, segundo os entrevistados.
As práticas de estratégia ainda estão em nível básico, e os processos, em implantação. A empresa possui seis sócios, e um Conselho foi instituído desde o início dessa sociedade. $O$ Conselho ainda não possui membros externos, tratando mais de questões operacionais do que de assuntos ligados aos stakeholders. O processo decisório acontece em reunião operacional com todo o staff, em que são discutidas ocorrências e maneiras de endereçá-las. As áreas têm autonomia relativa para fazer contratações ou aquisições. Segundo o Diretor Clínico: “A gente ainda não definiu números, por exemplo, aquisição, até tal valor, você tem autonomia para comprar, passou disso, você tem que ter a anuência do Diretor Financeiro, mas vamos definir esse processo. Ainda é um processo, como a gente tem uma casa pequena, todo mundo palpita, o Diretor Financeiro palpita em praticamente tudo..." Existe um business plan para os próximos 5 anos.

Em função da experiência do Diretor Financeiro e de alguns dos sócios investidores, há um forte suporte na área financeira da empresa. Mensuram resultados por paciente, custo do serviço prestado, custo fixo, margens, compras de material, medicamentos, custos dos serviços profissionais, dos serviços da equipe multifuncional, entre outros. Falta, no entanto, refinar esse processo para entender melhor, por exemplo, a diferença entre um paciente de média complexidade e o de alta complexidade, em termos de rentabilidade. Ou então estabelecer uma relação entre margem e origem do paciente em função da operadora, já que cada uma tem pacote de precificação diferente. Realizam um orçamento detalhado, que é acompanhado periodicamente.

(continua) 
Tabela 2 (continuação)

\begin{tabular}{|c|c|c|c|}
\hline Área & Hospital A * & Hospital B ** & Hospital C *** \\
\hline Marketing & 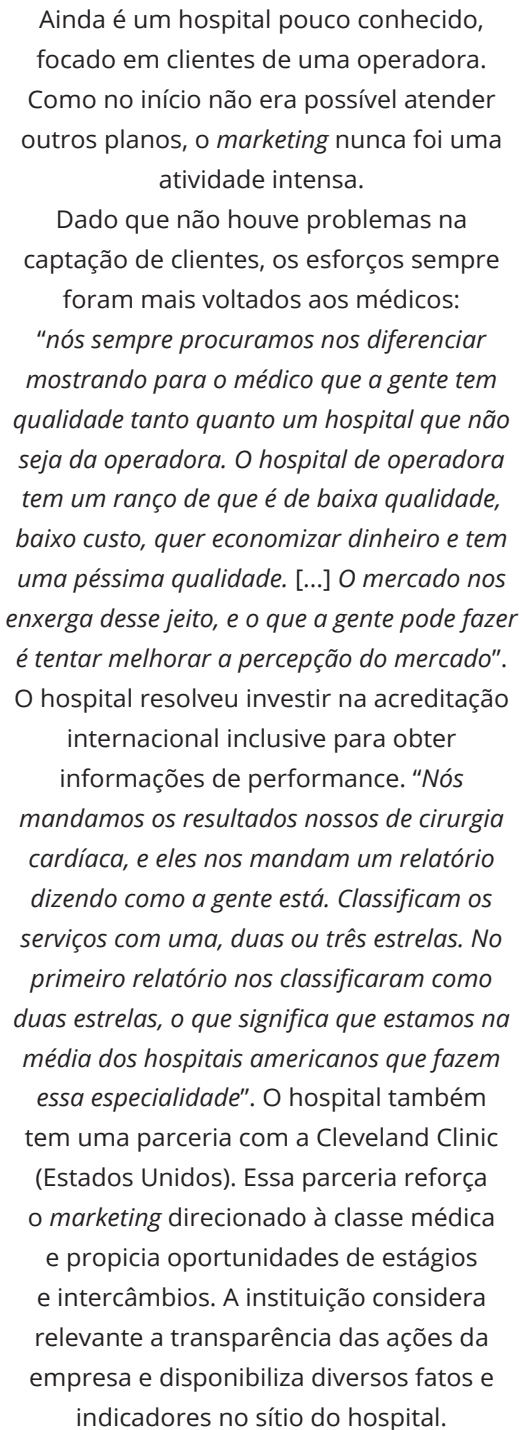 & $\begin{array}{c}\text { A área de marketing não costuma coletar } \\
\text { dados do mercado e dos concorrentes. } \\
\text { Trabalham sobre uma percepção genérica, } \\
\text { principalmente pelos dados da procura } \\
\text { ou do comportamento dos usuários dos } \\
\text { serviços do hospital. Seus dirigentes } \\
\text { entendem que a estratégia de marketing } \\
\text { deve ser pautada na diferenciação, mas } \\
\text { não há pesquisas para fundamentar } \\
\text { esse entendimento. Em relação aos } \\
\text { concorrentes, o hospital se destaca pela } \\
\text { gestão profissionalizada, com impacto } \\
\text { na qualidade dos seus serviços. A origem } \\
\text { do hospital, em empresas de consultoria } \\
\text { hospitalar, foi relevante para a consolidação } \\
\text { dessa característica. }\end{array}$ & 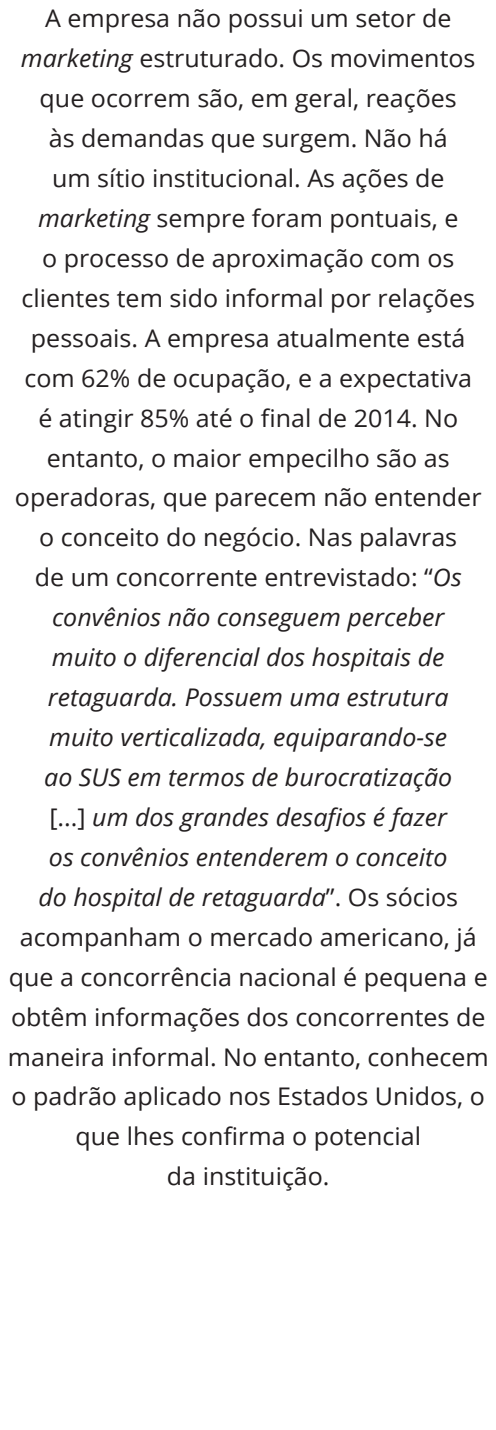 \\
\hline
\end{tabular}

\footnotetext{
* 400 funcionários, 90 leitos;

** 541 funcionários, 150 leitos;

*** 70 funcionários, 47 leitos.
} 
Tabela 3

Características das práticas de gestão.

\begin{tabular}{|c|c|c|c|c|}
\hline Práticas & Mínimo & Máximo & Média & Desvio padrão \\
\hline \multicolumn{5}{|l|}{ Recursos humanos } \\
\hline Equipes & 1 & 5 & 3,62 & 1,03 \\
\hline Gestão de pessoas & 1 & 5 & 3,28 & 1,33 \\
\hline Clima de trabalho & 2 & 5 & 3,75 & 1,09 \\
\hline Retenção de talentos & 1 & 5 & 2,98 & 1,32 \\
\hline Flexibilidade na organização & 1 & 5 & 4,00 & 1,10 \\
\hline Clareza de responsabilidades & 2 & 5 & 3,57 & 1,20 \\
\hline \multicolumn{5}{|l|}{ Marketing } \\
\hline Foco no cliente & 1 & 5 & 3,94 & 1,01 \\
\hline Monitoramento do mercado & 1 & 5 & 3,68 & 1,32 \\
\hline \multicolumn{5}{|l|}{ Operações } \\
\hline Fluxo do trabalho & 1 & 5 & 3,79 & 1,21 \\
\hline Melhoria contínua & 2 & 5 & 4,21 & 0,93 \\
\hline Monitoramento do desempenho & 2 & 5 & 3,53 & 1,12 \\
\hline Gestão à vista/Transparência de dados & 1 & 5 & 3,45 & 1,25 \\
\hline Solução de problemas & 1 & 5 & 3,51 & 1,08 \\
\hline Padronização & 1 & 5 & 3,74 & 1,22 \\
\hline Protocolos & 2 & 5 & 3,75 & 1,01 \\
\hline \multicolumn{5}{|l|}{ Finanças } \\
\hline Gestão financeira & 1 & 5 & 4,00 & 1,21 \\
\hline Gestão de custos & 1 & 5 & 3,80 & 1,26 \\
\hline \multicolumn{5}{|l|}{ Estratégia } \\
\hline Planejamento estratégico & 1 & 5 & 3,48 & 1,35 \\
\hline Inovação & 1 & 5 & 3,68 & 1,12 \\
\hline Média das práticas & 2,16 & 5 & 3,67 & 0,87 \\
\hline
\end{tabular}

Tabela 4

Perfil dos hospitais pesquisados na análise quantitativa.

\begin{tabular}{lc}
\hline Número de leitos (média) & 141 \\
Número de leitos (mediana) & 129 \\
Leitos de UTI (\%) & 15,5 \\
Hospitais especializados (\%) & 19,1 \\
Hospitais gerais (\%) & 80,9 \\
Hospitais com acreditação (\%) & 59,6 \\
Número de respondentes & 47 \\
\hline
\end{tabular}


O tamanho da amostra dificulta análises mais complexas. Empregou-se a abordagem de agrupamentos, utilizando a técnica de K-means do IBM SPSS 21. Encontrou-se a melhor solução para três agrupamentos que foram chamados: Básico (30\% dos hospitais) para o grupo com menor nível de utilização das práticas; Intermediário (28\% dos hospitais) para o grupo com nível médio de utilização das práticas e Excelência (34\% dos hospitais) para o grupo com nível avançado de utilização das práticas. Esses agrupamentos foram gerados pelos escores de cada prática e representam grupos relativamente homogêneos quanto às práticas de gestão. Os resultados dos centroides finais dos agrupamentos e as características dos hospitais de cada agrupamento estão apresentados na Tabela 5.

Pela Tabela 5, verifica-se que a adoção das práticas está bastante relacionada ao porte do hospital, dado que quanto maior o nível de utilização das práticas, maior é seu tamanho. Número de internações e número de cirurgias também podem ser considerados como variáveis proxies para o tamanho do hospital. No entanto, não foi encontrada relação entre adoção de práticas e número de leitos de UTI.

Em relação aos indicadores de desempenho, percebe-se uma relação entre a adoção das práticas e o melhor desempenho. É o caso dos indicadores internações por leito, taxa de ocupação ou ainda percentual com acreditação, claramente superior às médias nacionais. Tais indicadores são muito relevantes para se avaliar a rentabilidade da operação, sugerindo que as práticas de gestão trazem um benefício para a efetividade deles e, comparativamente, entre os hospitais, uma maior capacidade competitiva. Estudos anteriores em hospitais encontraram resultados similares. Bloom et al. 46 também acharam um efeito positivo de práticas de gestão no desempenho. Li et al. 47 sugerem a associação entre porte e uma gestão mais sofisticada, com maior conteúdo estratégico e, consequentemente, melhor desempenho para hospitais.

Nota-se que as diferenças do indicador permanência média (em dias) não se mostraram significativas nos testes post hoc.

\section{Considerações finais}

Tendo como propósito identificar os efeitos das práticas tradicionais de gestão no desempenho em hospitais privados de médio porte, esta pesquisa contribui ao caracterizar a utilização das principais práticas gerenciais de operações, recursos humanos, estratégia, marketing e finanças e classificar os hospitais por nível de utilização dessas práticas. Também evidencia as práticas de gestão mais utilizadas por área funcional e a relação entre o nível de utilização dessas práticas e o desempenho médio dos hospitais.

Constata-se que existe uma grande dispersão no nível de adoção de práticas gerenciais básicas na amostra estudada. Entre os respondentes, há hospitais com um nível competente e atualizado na aplicação de práticas, enquanto outros carecem de sistemas básicos de práticas consagradas como gestão financeira, gestão de custos e planejamento estratégico. É surpreendente observar que alguns hospitais afirmaram que "praticamente não utilizam" práticas de gestão financeira.

Outra constatação é a relação entre a adoção das práticas e o desempenho dos hospitais. Os que apresentavam maior grau de adoção de práticas apresentaram maior taxa de ocupação, internações por leito e certificados de acreditação. Tal resultado pode ser um incentivo para que outros hospitais também adotem ou aperfeiçoem suas práticas de gestão. É possível também extrapolar os resultados encontrados e sugerir que outros hospitais privados de maior porte ou hospitais públicos possam se beneficiar da implementação de práticas de gestão em suas atividades.

A fase qualitativa, realizada junto a serviços que reconhecidamente utilizam instrumentos de gestão, indica que novas práticas estão sendo implantadas ou têm sua implantação considerada pelos hospitais. Isso é explicado pela recente abordagem da profissionalização da administração no setor, mais encontrada em países como Estados Unidos, Reino Unido, Canadá e França, nos últimos dez anos 48. A fase quantitativa, apesar da limitada amostra, indica que o nível de adoção de práticas parece estar associado ao tamanho do hospital. Conforme esperado, os maiores têm um melhor nível de gestão. A relação com o porte foi encontrada em estudos anteriores nos Estados Unidos e no Brasil 49,50,51. O mais amplo uso de práticas também está associado a um desempenho superior, pelo menos em termos de taxa de utilização. Embora a fase quantitativa não permita uma clara diferenciação quanto à utili- 


\section{Tabela 5}

Resultados dos agrupamentos.

\begin{tabular}{|c|c|c|c|}
\hline Variáveis & Excelência * & Intermediário ** & Básico *** \\
\hline \multicolumn{4}{|l|}{ Hospitais } \\
\hline Número de hospitais participantes & 16 & 13 & 14 \\
\hline Amostra (\%) & 34,0 & 28,0 & 30,0 \\
\hline \multicolumn{4}{|l|}{ Práticas } \\
\hline \multicolumn{4}{|l|}{ Recursos humanos } \\
\hline Equipes & 4,5 & 3,5 & 2,7 \\
\hline Gestão de pessoas & 4,4 & 3,5 & 1,9 \\
\hline Clima de trabalho & 4,5 & 4,0 & 2,7 \\
\hline Retenção de talentos & 4,3 & 2,9 & 1,6 \\
\hline Flexibilidade na organização & 4,1 & 4,5 & 3,2 \\
\hline Clareza de responsabilidades & 4,8 & 3,3 & 2,4 \\
\hline \multicolumn{4}{|l|}{ Marketing } \\
\hline Foco no cliente & 4,7 & 3,6 & 3,2 \\
\hline Monitoramento do mercado & 4,8 & 3,0 & 3,1 \\
\hline \multicolumn{4}{|l|}{ Operações } \\
\hline Fluxo do trabalho & 4,8 & 3,1 & 3,1 \\
\hline Melhoria contínua & 5,0 & 4,1 & 3,4 \\
\hline Monitoramento do desempenho & 4,7 & 2,9 & 2,6 \\
\hline Gestão à vista/Transparência de dados & 4,6 & 3,2 & 2,4 \\
\hline Solução de problemas & 4,5 & 3,2 & 2,5 \\
\hline Padronização & 4,8 & 3,5 & 2,8 \\
\hline Protocolos & 4,6 & 3,8 & 2,7 \\
\hline \multicolumn{4}{|l|}{ Finanças } \\
\hline Gestão financeira & 5,0 & 3,5 & 3,2 \\
\hline Gestão de custos & 4,8 & 3,2 & 3,0 \\
\hline \multicolumn{4}{|l|}{ Estratégia } \\
\hline Planejamento estratégico & 4,8 & 2,8 & 2,4 \\
\hline Inovação & 4,8 & 3,2 & 2,9 \\
\hline Média das práticas & 4,7 & 3,4 & 2,7 \\
\hline \multicolumn{4}{|l|}{ Perfil dos hospitais } \\
\hline Leitos & 210 & 133 & 90 \\
\hline Leitos de UTI (\%) & 24,7 & 14,1 & 22,5 \\
\hline Funcionários assistenciais & 600 & 488 & 207 \\
\hline Internações & 13.800 & 7.918 & 4.475 \\
\hline Cirurgias & 7.223 & 6.897 & 5.817 \\
\hline \multicolumn{4}{|l|}{ Indicadores } \\
\hline Internações por leito & 65,7 & 59,5 & 49,7 \\
\hline Taxa de ocupação (\%) & 80,3 & 73,6 & 55,9 \\
\hline Permanência média em dias & 9,4 & 5,3 & 9,9 \\
\hline Com acreditação (\%) & 93,8 & 46,2 & 42,9 \\
\hline
\end{tabular}

* Grupo de hospitais com nível avançado de utilização de práticas;

** Grupo de hospitais com nível médio de utilização de práticas;

*** Grupo de hospitais com nível baixo de utilização de práticas. 
zação de práticas, o estudo qualitativo sugere que as práticas de marketing e planejamento estratégico são as mais embrionárias, enquanto as gestões de operações e finanças parecem mais desenvolvidas.

De forma geral, os resultados indicam que as práticas básicas de gestão, embora conhecidas e divulgadas, podem sim trazer diferenças de desempenho, oferecendo suporte à nova perspectiva da PBV 11. A sua não aplicação por hospitais de médio porte traz uma limitação de capacidade competitiva que pode ser entendida como mais um componente do custo Brasil, mas, dessa vez, um componente interno. $\mathrm{O}$ aparente paradoxo é que não há barreiras de conhecimento e tecnologia para explicar essa limitação. As práticas analisadas são elementares, contidas na formação básica de administração. $\mathrm{Na}$ abordagem da visão baseada em recursos, elas são competências facilmente imitáveis. Se esses hospitais não as utilizam, talvez existam novos tipos de barreiras de natureza cognitiva e comportamental nesse contexto, que podem ser exploradas em futuras pesquisas. A constatação da situação e o estudo dessas potenciais barreiras podem constituir-se numa contribuição prática relevante para a competitividade da média empresa brasileira e para a melhoria de serviços fundamentais como o de saúde.

Como limitação do estudo, pode-se apontar a amostra reduzida, que não permitiu a aplicação de testes estatísticos mais robustos. Além disso, a amostra se limitou ao Estado de São Paulo e seria interessante verificar também a adoção de tais práticas no restante do país.

Em relação aos estudos futuros no tema, sugere-se que a pesquisa que originou este artigo seja continuada, a fim de se considerar os efeitos longitudinais da utilização das práticas de gestão nos hospitais de médio porte. À medida que esses hospitais dominam sua utilização, podem ser estudados os tipos de aperfeiçoamentos que são aplicados, a intensidade e o impacto da aplicação dos indicadores derivados dessas práticas para a gestão cotidiana do hospital. Pode-se pensar também na possibilidade de se analisar outras práticas que os hospitais estejam utilizando e que não constam da relação utilizada no trabalho. Dessa forma, espera-se contribuir, ainda que marginalmente, para a melhora da qualidade dos serviços hospitalares no Brasil.

\section{Colaboradores}

L. A. L. Brito, A. M. Malik, E. Brito, S. Bulgacov e T. Andreassi contribuíram na coleta, análise e interpretação de dados; redação e revisão final do manuscrito.

\section{Referências}

1. La Forgia GM, Couttolenc BF. Desempenho hospitalar brasileiro: em busca da excelência. São Paulo: Singular; 2009.

2. Lima SML, Barbosa PR, Portela MC, Ugá MAD, Vasconcellos MM, Gerschman S. Caracterização gerencial dos hospitais filantrópicos no Brasil. Cad Saúde Pública 2004; 20:1249-61.

3. Sediyama MYN, Aquino ACB, Bonacim CAG. Avaliação da eficiência de hospitais filantrópicos de pequeno porte pela Análise Envoltória de Dados (DEA). In: Anais do Encontro de Administração Pública e Governo 2012. Rio de Janeiro: Associação Nacional de Pós-Graduação e Pesquisa em Administração; 2012. p. 1-16.

4. Newbert SL. Empirical research on the resource-based view of the firm: an assessment and suggestions for future research. Strategic Management Journal 2007; 28:121-46.

5. Barney JB. Looking inside for competitive advantage. Academy of Management Executive 1995; 9:49-61.

6. McGahan AM, Porter ME. How much does industry matter, really? Strategic Management Journal 1997; 18:15-30. 
7. Brito LAL, Vasconcelos FC. The variance composition of firm growth rates. Brazilian Administration Review 2009; 6:118-36.

8. Vecina Neto G, Malik A. Gestão em saúde. Rio de Janeiro: Editora Guanabara Koogan; 2011.

9. Bloom N, Reenen JV. Measuring and explaining management practices across firms and countries. QJ Econ 2007; 122:1351-408.

10. Bloom N, Reenen JV. Why do management practices differ across firms and countries? J Econ Perspect 2010; 24:203-24.

11. Bromiley P, Rau D. Towards a practice-based view of strategy. Strategic Management Journal 2014; 35:1249-56.

12. Queiroz, ACS, Albuquerque LG, Malik AM. Gestão estratégica de pessoal e inovação: estudos de caso no contexto hospitalar. Revista de Administração (São Paulo) 2013; 48:658-70.

13. Rogers EM. Diffusion of innovations. 5th Ed. New York: Free Press; 2003.

14. World Health Organization. World Health Statistics 2012. Part III: global health indicators. http://www.who.int/healthinfo/ EN_WHS2012_Part3.pdf (acessado em 30/ Nov/2012).

15. Simon H. Campeãs ocultas. Porto Alegre: Editora Bookman; 2003.

16. Cleverley WO, Harvey R. Competitive strategy for successful hospital management. Hosp Health Serv Adm 1992; 37:53-68.

17. Qin Y. Making innovation in management and formulation of competitive strategies of the hospital. Academic Journal of PLA Postgraduate Medical School 2006; (2):1-15.

18. Pereira SR, Paiva PB, Souza PRS, Siqueira G, Pereira AR. Sistemas de informações para a gestão hospitalar. J Health Inform 2012; 4:1-15.

19. Souza AA, Guerra M, Lara CO, Gomide PLR, Pereira CM, Freitas DA. Controle de gestão em organizações hospitalares. REGE - Revista de Gestão da USP 2009; 16:15-29.

20. Barney JB, Clark DN. Resource-based theory. New York: Oxford University Press; 2007.

21. Wernerfelt B. A resource-based view of the firm. Strategic Management Journal 1984; 5:171-80.

22. Barney JB. Firm resources and sustained competitive advantage. J Manage 1991; 17:99-120.

23. Peteraf MA. The cornerstones of competitive advantage: a resource-based view. Strategic Management Journal 1993; 14:179-91.

24. Barney JB, Ketchen DJ, Wright M. The future of resource-based theory: revitalization or decline? J Manage 2011; 37:1299-315.

25. Vorhies DW, Morgan NA. Benchmarking marketing capabilities for sustainable competitive advantage. J Mark 2005; 69:80-94.

26. Oyedijo A, Idris AA, Aliu AA. Impact of marketing practices on the performance of small business enterprises: empirical evidence from $\mathrm{Ni}$ geria. European Journal of Economics, Finance and Administrative Sciences 2012; 46:130-46.

27. Coviello NE, Brodie RJ, Munro HJ. An investigation of marketing practice by firm size. Journal of Business Venturing 2000; 15:523-45.
28. Ellis PD. Market orientation and marketing practice in a developing economy. Eur J Mark 2005; 39:629-45.

29. Webster Jr. FE. The rediscovery of the marketing concept. Bus Horizons 1988; 31:29-39.

30. Butcher L. How to create exceptional patient experiences: without exception. Hosp Health Netw 2015; 2 Dec. http://www.hhnmag.com/ articles/6702-how-to-create-exceptional-pa tient-experiences-without-exception.

31. Burns J. Chief experience officers push patients to forefront. Manag Care 2015; 24:51-3.

32. SAX Customer Experience Research \& Consulting. O que está na agenda dos hospitais? Melhores Práticas 2015; 15:36-41.

33. Stewart M, Brown JB, Donner A, McWhinney IR, Oates J, Weston WW, et al. The impact of patient-centered care on outcomes. J Fam Pract 2000; 49:796-804.

34. Núcleo Técnico da Política Nacional de Humanização, Ministério da Saúde. HumanizaSUS: Política Nacional de Humanização. A humanização como eixo norteador das práticas de atenção e gestão em todas as instâncias do SUS. Brasília: Ministério da Saúde; 2004. (Série B. Textos Básicos de Saúde).

35. Moore M, Fairhurst A. Marketing capabilities and firm performance in fashion retailing. Journal of Fashion Marketing and Management: An International Journal 2003; 7:386-97.

36. Morgan NA, Vorhies DW, Mason CH. Market orientation, marketing capabilities and firm performance. Strategic Management Journal 2009; 30:909-20.

37. Slater SF, Narver JC. Customer-led and market-oriented: Let's not confuse the two. Strategic Management Journal 1998; 19:1001-6.

38. Spillan J, Parnell J. Marketing resources and firm performance among SMEs. European Management Journal 2006; 24:236-45.

39. Bartlett CA, Ghoshal S. Going global: lessons from late movers. Harv Bus Rev 2000; 78:132-42.

40. Ward PT, Leong GK, Boyer KK. Manufacturing proactiviness and performance. Decision Sciences $1994 ; 25: 337-58$.

41. Hausman WH, Montgomery DB, Roth AV. Why should marketing and manufacturing work together? Some exploratory empirical results. Journal of Operations Management 2002; 20:241-57.

42. Advisory Board Company. Next-generation capacity management collaborating for clinically appropriate and efficient inpatient throughput. Washington DC: Advisory Board Company; 2010.

43. Donaire DA. Utilização do estudo de casos como método de pesquisa na área da administração. Revista IMES 1997; 40:9-19.

44. Ketokovi M, Choi T. Renaissance of case research as a scientific method. Journal of Operations Management 2014; 32:232-40.

45. Richardson RJ. Pesquisa social: métodos e técnicas. 3a Ed. São Paulo: Editora Atlas; 1999. 
46. Bloom N, Propper C, Seiler S, Van Reenen J. The impact of competition on management quality: evidence from public hospitals. Rev Econ Stud 2015; 82:457-89.

47. Li LX, Benton WC, Leong GK. The impact of strategic operations management decisions on community hospital performance. Journal of Operations Management 2002; 20:389-408.

48. Griffith JR, Pattullo A, Alexander JA, Jelinek RC, Foster DA. Is anybody managing the store? National trends in hospital performance. J Healthc Manag 2006; 51:392-405.

\begin{abstract}
Traditional management practices are sometimes considered merely a necessary condition for superior performance. Other resources and competencies with higher barriers to imitation are assumed to be potential sources of competitive advantage. This study describes and analyzes the effect of traditional management practices on the performance of medium-sized hospitals. Medium-sized companies frequently display the greatest differences in management practices, and only recently did the hospital sector seek ways to develop its competitiveness in the administrative arena. The results generally indicate that basic management practices can make differences in performance, offering support for the new practice-based view $(P B V)$. Hospitals with the highest rate of adoption of practices had the highest occupancy rate, hospital-bed admissions, and accreditation. Lack of adoption of management practices by mediumsized hospitals limits their competitive capacity and can be viewed as a component of the so-called Brazil cost, but in this case an internal component.
\end{abstract}

Health Services Administration; Health Management; Hospitals
49. Goldstein SM, Ward PT, Leong GK, Butler TW. The effect of location, strategy, and operations technology on hospital performance. Journal of Operations Management 2002; 20:63-75.

50. Correa LRC. Os hospitais de pequeno porte do Sistema Único de Saúde e a segurança do paciente [Dissertação de Mestrado]. São Paulo: Escola de Administração de Empresas de São Paulo, Fundação Getúlio Vargas; 2009.

51. Ugá MAD, López EM. Os hospitais de pequeno porte e sua inserção no SUS. Ciênc Saúde Coletiva $2007 ; 12: 915-28$.

\section{Resumen}

Las prácticas tradicionales de gestión se consideran a veces una condición meramente necesaria para un cometido superior. Otros recursos $y$ competencias con mayores dificultades de ser susceptibles de imitación son las que serían los potenciales aspectos que suponen una ventaja competitiva frente a la competencia. Este estudio describe y analiza el efecto de prácticas tradicionales de gestión en el desempeño de hospitales de tamaño medio. Las empresas de tamaño medio son aquellas en las que es habitual encontrar mayores diferencias en el uso de las prácticas, y el sector hospitalario sólo ha buscado recientemente, en el área de administración, formas de desarrollar su competitividad. De manera general, los resultados indican que las prácticas básicas de gestión sí pueden conllevar diferencias de desempeño, ofreciendo apoyo a una nueva perspectiva de la PBV (practice-based view). De hecho, se verificó que los hospitales que presentaron una mayor tasa de adopción de prácticas, poseían una mayor tasa de ocupación, internamientos por cama y certificado de acreditación. La no adopción de las prácticas por parte de los hospitales de tamaño medio conlleva una limitación de su capacidad competitiva, que puede ser entendida como un componente más del coste Brasil, sin embargo, esta vez, como un componente interno.

Administración de los Servicios de Salud;

Gestión en Salud; Hospitales
Recebido em 27/Fev/2015

Versão final reapresentada em 13/Abr/2016

Aprovado em 12/Mai/2016 